\title{
A assistência ao parto normal e a satisfação das puérperas
}

RESUMO | Objetivo: Averiguar o que diz a literatura quanto à satisfação das puérperas em relação à assistência recebida no trabalho de parto e parto nos serviços de saúde. Método: Estudo qualitativo do tipo revisão integrativa, nas Bases de Dados: MEDLINE/BVS, SciELO, BDENF, PubMed, e DOAJ, no período de 15 de janeiro a 20 de fevereiro de 2021. Foi realizada leitura analítica dos estudos identificando os pontos chave para hierarquização e síntese das ideias. Resultados: Foram selecionados 14 estudos. Os dados obtidos com a pesquisa apontam para a inexistência de unanimidade quanto à percepção e satisfação das mulheres em relação à assistência ao trabalho de parto e parto. Conclusão: Portanto, atenção, acolhimento, cuidado e vínculo são elementos fundamentais para uma melhor assistência e, consequentemente, para o alcance da satisfação destas mulheres em relação à assistência recebida.

Descritores: Satisfação do Paciente; Parto Normal; Pós-Parto

ABSTRACT | Objective: To investigate what the literature says about the satisfaction of postpartum women in relation to the assistance received in labor and delivery in health services. Method: Qualitative study of the integrative review type, in Databases: MEDLINE/BVS, SciELO, BDENF, PubMed, and DOAJ, from January 15th to February 20th of 2021. An analytical reading of the studies was carried out, identifying the key points for the hierarchy and synthesis of ideas. Results: 14 studies were selected. The data obtained from the survey point to the lack of unanimity regarding the perception and satisfaction of women in relation to care during labor and delivery. Conclusion: Therefore, attention, reception, care and bonding are fundamental elements for better care and, consequently, for achieving these women's satisfaction in relation to the care received.

Descriptors: Patient Satisfaction; Natural Childbirth; Postpartum Period.

RESUMEN | Objetivo: Investigar lo que dice la literatura sobre la satisfacción de las mujeres posparto en relación a la asistencia recibida en el trabajo de parto y parto en los servicios de salud. Método: Estudio cualitativo del tipo revisión integrativa, en Bases de datos: MEDLINE / BVS, SciELO, BDENF, PubMed, y DOAJ, del 15 de enero al 20 de febrero de 2021. Análisis analítico Se realizó la lectura de los estudios, identificando los puntos clave para la jerarquía y síntesis de las ideas. Resultados: Se seleccionaron 14 estudios. Los datos obtenidos de la encuesta apuntan a la falta de unanimidad en cuanto a la percepción y satisfacción de las mujeres en relación al cuidado durante el trabajo de parto y parto. Conclusión: Por lo tanto, la atención, la recepción, el cuidado y la vinculación son elementos fundamentales para un mejor cuidado y, en consecuencia, para lograr la satisfacción de estas mujeres en relación con el cuidado recibido.

Descriptores: Satisfacción del Paciente; Parto Normal; Periodo Posparto

Magda Kelanny de Oliveira dos Santos

Especialista em Enfermagem Obstétrica na modalidade Residência pela Escola de Saúde Pública de Pernambuco (ESPPE).

ORCID: 0000-0002-9802-8428

\section{Liniker Scolfild Rodrigues da Silva}

Especialista em Enfermagem Obstétrica na modalidade Residência pela Faculdade de Enfermagem Nossa Senhora das Graças/Universidade de Pernambuco (FENSG/ UPE). Sanitarista, Especialista em Saúde Coletiva na modalidade Residência pela Faculdade de Ciências Médicas (FCM)/UPE. Recife, Pernambuco (PE), Brasil

ORCID: 0000-0003-3710-851X

\section{Raquel Bezerra dos Santos}

Mestre em Ciências da Saúde pela Universidade Federal de Pernambuco (UFPE). Recife, Pernambuco (PE), Brasil. ORCID: 0000-0002-9730-4718

\section{Beatriz Raquel Lira da Fonsêca}

Discente do Curso de Bacharel em Enfermagem pela Faculdade de Enfermagem Nossa Senhora das Graças/Universidade de Pernambuco (FENSG/UPE). Recife, Pernambuco (PE), Brasil.

ORCID: 0000-0002-4430-2479

\section{Aline Barros de Oliveira}

Residente no Programa de Residência em Enfermagem Obstétrica na modalidade Residência pela Escola de Saúde Pública de Pernambuco (ESPPE). Recife, Pernambuco (PE), Brasil.

ORCID: 0000-0003-0427-7181

\section{Mônica Josefa da Silva Oliveira}

Residente no Programa de Residência em Enfermagem Obstétrica na modalidade Residência pela Escola de Saúde Pública de Pernambuco (ESPPE). Recife, Pernambuco (PE), Brasil.

ORCID: 0000-0003-2395-298X
Recebido em: 25/08/2021

Aprovado em: 12/11/202

INTRODUÇÃO<smiles>C1CCC2CCCCC2CC1</smiles>
parto e o nascimento de um filho são considerados alguns dos acontecimentos mais im portantes na vida de uma mulher. Com o decorrer do tempo, ambos têm passado por diversas transformações, uma delas é a transição do parto domiciliar para o parto hospitalar. ${ }^{(1)}$ Pensando em uma melhor assistência às parturientes, a Organização Mundial de Saúde (OMS) e o Programa de Humanização no Pré-Natal e Nascimento (PHPN) estabelecem um novo modelo de assistência obstétrica, na qual caracteriza a mulher como protagonista do processo de parir, valorizando uma assistência que proporciona um 
atendimento de qualidade, articulando um acolhimento desde o pré-natal até o nascimento. (1)

A fim de garantir uma assistência obstétrica integral à mulher e preservar seus direitos de escolha, bem como à criança o direito ao nascimento seguro e ao crescimento e desenvolvimento saudáveis, o Ministério da Saúde (MS) instituiu, também, a estratégia Rede Cegonha em 2011. (2)

Atualmente, métodos não convencionais estão sendo cada vez mais utilizados e a proposta é a humanização na assistência ao parto até o nascimento. Estudos evidenciam que a utilização desses métodos não farmacológicos proporciona à mulher um maior protagonismo no trabalho de parto fazendo com que o tempo seja inferior, dando conforto, causando menos danos, reduzindo a necessidade de analgesias, fazendo com que a experiência seja positiva e o parto aconteça espontaneamente. (3-4)

Humanizar o parto não significa apenas prestar assistência ao parto normal, realizar ou não procedimentos, mas sim tornar a mulher protagonista e não apenas espectadora desse momento, dando-lhe liberdade de escoIha nos processos decisórios. O parto humanizado inclui o respeito ao processo fisiológico e à dinâmica de cada nascimento, nos quais as intervenções devem ser cuidadosas, evitando os excessos e visando sempre a promoção do parto e nascimento saudáveis. (5)

Tendo em conta que a humanização do parto não tem o enfoque somente técnico, mas também deve ser associada ao respeito dos direitos da paciente, a satisfação com o atendimento recebido é uma forma indireta de identificar a qualidade dos serviços de saúde.

Diante disso, com o intuito de subsidiar a reflexão acerca da assistência ao parto normal sob a perspectiva da mulher, este estudo tem como objetivo averiguar o que diz a literatura quanto à satisfação das puérperas em relação

66

A fim de garantir uma assistência obstétrica integral à mulher e preservar seus direitos de escolha, bem como à criança o direito ao nascimento seguro e ao crescimento e desenvolvimento saudáveis, o Ministério da Saúde (MS) instituiu, também, a estratégia Rede Cegonha em 2011 à assistência recebida no trabalho de parto e parto nos serviços de saúde.

MÉTODO

Trata-se de uma revisão integrativa na qual foram percorridas as seguintes etapas: elaboração da questão norteadora e objetivo do estudo; definição dos critérios de inclusão e exclusão das produções científicas; busca de estudos científicos em bases de dados e bibliotecas virtuais; análise e categorização dos estudos selecionados; interpretação dos resultados e discussão dos achados.

Para o levantamento da questão norteadora, utilizou-se a estratégia PICo. A estratégia PICo representa um acrônimo para $\mathrm{P}$ - população/paciente, 1 - intervenção/interesse, Co - comparação/ausência e Outcomes (Desfecho), e pode ser utilizada para construir questões de pesquisa de naturezas diversas, de maneira adequada, com foco no escopo da pesquisa e a fim de evitar a realização de buscas desnecessárias. (6)

Fazendo-se valer desta estratégia, definiu-se para o presente estudo a estratégia PICo (P: Puérperas; I: Satisfação da assistência recebida; Co: Trabalho de parto e Parto). Desta forma, definiu-se a seguinte questão norteadora da pesquisa: "O que diz a literatura quanto à satisfação das puérperas em relação à assistência recebida no trabaIho de parto e parto?".

Para seleção dos artigos, utilizaram-se como critérios de inclusão: ser artigo original, disponíveis na íntegra, no idioma português ou espanhol, publicados nos últimos 10 anos, e que respondessem ao objetivo do estudo. Foram excluídos: literaturas cinzas, bem como publicações repetidas de estudos em mais de uma base de dados os artigos que não responderam à questão norteadora do estudo. A delimitação

$\int$ temporal deu-se em razão do redesenho na política de saúde da mulher por 
meio da portaria $n^{\circ} 1.459$ de 24 de junho de 2011, na qual o Governo Federal instituiu, no âmbito do Sistema Único de Saúde (SUS), a Rede Cegonha.

O levantamento dos dados ocorreu entre os dias 15 de janeiro a 20 de fevereiro de 2021, nas seguintes Bases de Dados: Medical Literature Analysis and Retrieval System Online via Biblioteca Virtual da Saúde (MEDLINE/ BVS); Banco de Dados em Enfermagem (BDENF); PubMed; e Directory of Open Access Journals (DOAJ), bem como na Biblioteca Virtual, Scientific Electronic Library Online (SciELO).

Buscaram-se os artigos indexados a partir dos seguintes Descritores em Ciências da Saúde (DeCS), na Biblioteca Virtual de Saúde: "Satisfação do Paciente", "Parto Normal", "Assistência ao Parto" e "Puerpério", utilizando o operador booleano AND. (Quadro 1).

A seleção dos estudos baseou-se no Preferred Reporting Items for Systematic Review and Meta-Analyses (PRISMA) (7), com o objetivo de auxiliar no desenvolvimento de artigos. A princípio, eliminaram-se por meio da leitura de títulos e resumos, estudos que não se enquadraram nos critérios de inclusão, e estudos duplicados. Destes pré-selecionados, realizou-se leitura na íntegra, a fim de verificar os que atendem à questão norteadora e aos critérios de inclusão/exclusão. Construiu-se então a amostra final com estudos pertinentes aos critérios pré-estabelecidos (Figura 1).

Foram localizados 793 artigos, sendo 65 artigos disponíveis na MEDLINE/ BVS; 133 na SciELO; 440 na BDENF; 125 na PubMed e 30 na DOAJ. Após supressão de artigos duplicados, leitura do título e leitura do resumo foram excluídos 773 artigos, resultando em 20 artigos pré-selecionados para leitura na íntegra. Após a leitura na íntegra, a amostra final com estudos pertinentes aos critérios pré-estabelecidos foi de 14 artigos.

Após a leitura dos artigos selecio-

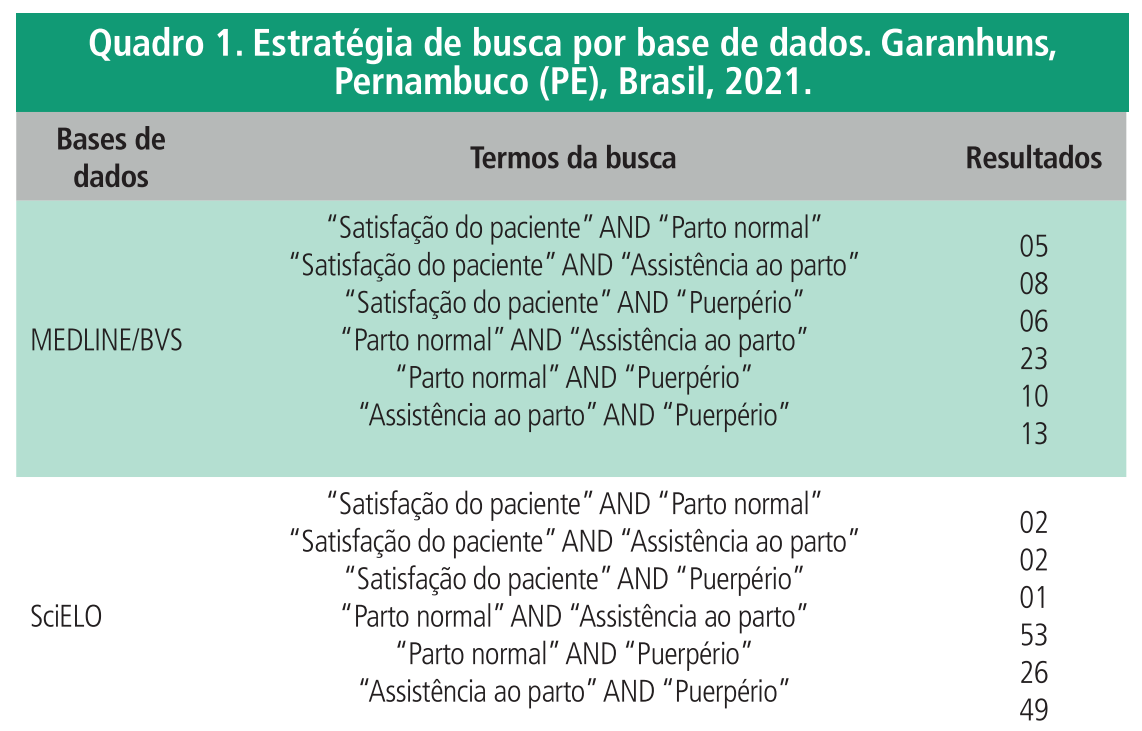

$\begin{array}{lcc} & \text { "Satisfação do paciente" AND "Parto normal" } & 16 \\ \text { "Satisfação do paciente" AND "Assistência ao parto" } & 28 \\ \text { "SDENF } & \text { "Patisfação do paciente" AND "Puerpério" } & 14 \\ & \text { "Parto normal" AND "Puerpério" } & 179 \\ \text { "Assistência ao parto" AND "Puerpério" } & 44 \\ & & 159 \\ \text { "Patient Satisfaction" AND "Natural Childbirth" } & \\ \text { "Patient Satisfaction" AND "Delivery assistance" } & 21 \\ \text { PubMed } & \text { "Patient Satisfaction" AND "puerperium" } & 5 \\ & \text { "Natural Childbirth" AND "Delivery assistance" } & 78 \\ \text { "Natural Childbirth" AND "puerperium" } & 3 \\ \text { "Delivery assistance" AND "puerperium" } & 15 \\ & & 3 \\ \text { DOA } & \text { "Patient Satisfaction" AND "Delivery assistance" } & 2 \\ \text { "Patient Satisfaction" AND "puerperium" } & 7 \\ \text { "Natural Childbirth" AND "Delivery assistance" } & 4 \\ \text { "Natural Childbirth" AND "puerperium" } & 8 \\ \text { "Delivery assistance" AND "puerperium" } & 3 \\ & \end{array}$

Fonte: Dados da pesquisa, 2021.

nados os estudos foram categorizados, classificando o conhecimento produzido em níveis de evidência de acordo com Melnyk \& Fineout-Overholt(8): Nível I - Revisão sistemática, metanálise ou diretrizes clínicas oriundas de revisões sistemáticas de ensaios clínicos randomizados e controlados; Nível II - Ensaio clínico randomizado controlado; Nível III - Ensaios clínicos bem delineados sem randomização; Nível
IV - Estudo de coorte e de caso-controle bem delineados; Nível V - Revisão sistemática, de estudos descritivos e qualitativos; Nível VI - Um único estudo descritivo ou qualitativo; e por fim, Nível VI - Opiniões de autoridades e/ou parecer de comissão de especialistas.

Por fim, obteve-se a sumarização das informações do corpus por meio de um instrumento: identificação do artigo original; autoria do artigo; ano de pu- 


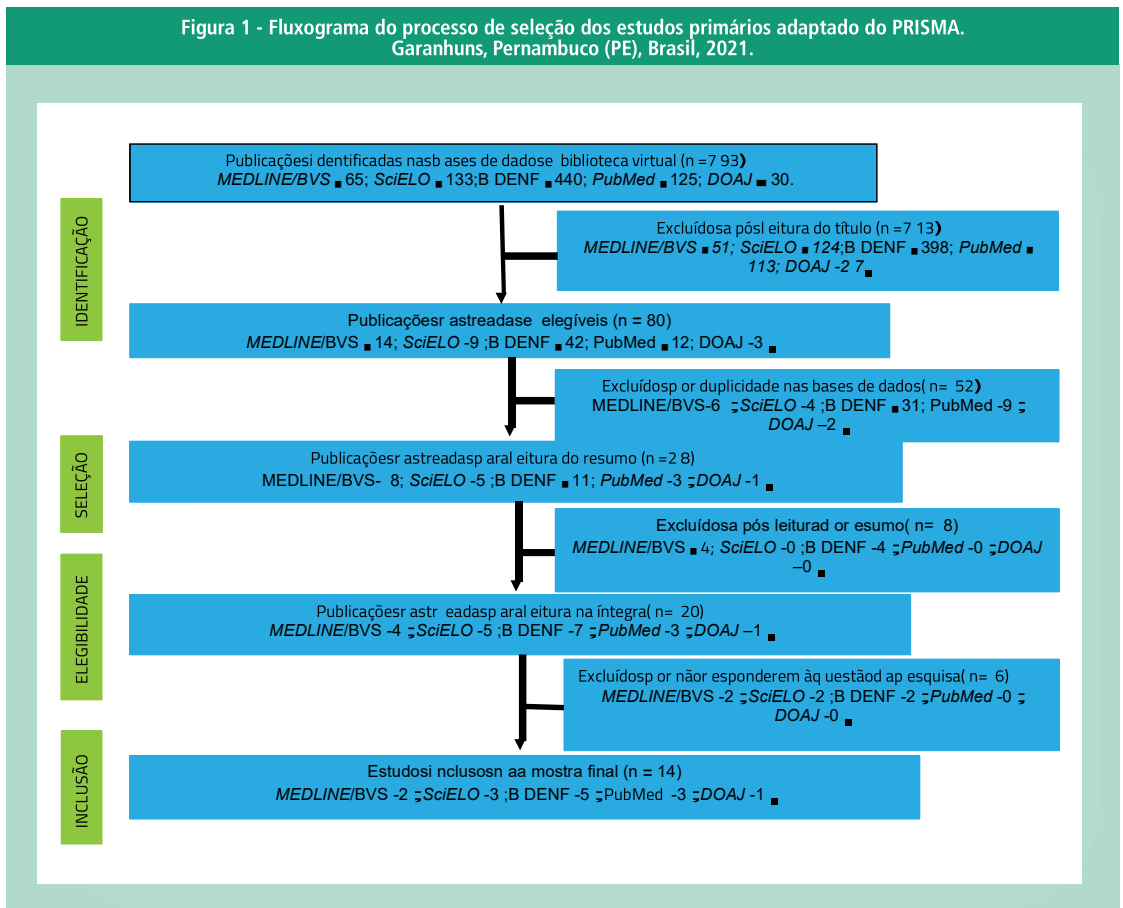

Fonte: Dados da pesquisa, 2021. blicação; país; características metodológicas do estudo; e amostra do estudo. Foi realizada leitura analítica dos estudos identificando os pontos chave para hierarquização e síntese das ideias.

Objetivando uma melhor compreensão e visualização dos principais achados, os dados foram organizados em quadros e tabelas, expostos de forma descritiva.

\section{RESULTADOS}

Para a visualização dos estudos que compuseram a amostra desta revisão integrativa, foi elaborada uma tabela evidenciando os resultados encontrados nos estudos de acordo seus títulos, autores, anos de publicação, níveis de evidências, objetivos e resultados. Em relação ao delineamento metodológico no tocante ao nível de evidência científica, constatou-se que a maioria

Tabela 1: Sintese dos principais achados quanto à satisfação das puérperas em relação à assistência recebida no trabalho de parto e parto nos serviços de saúde. Garanhuns, Pernambuco (PE), Brasil, 2021.

N Título/Base $\quad \begin{gathered}\text { Autores } \\ \text { (Ano) }\end{gathered} \quad$ País $\begin{gathered}\text { Nível de } \\ \text { Evidên- } \\ \text { cia }\end{gathered} \quad$ Objetivo

La comunicación y la satisfacción de las primíparas en un servicio público de salud. / MEDLINE/BVS

Díaz-Sáez,
Jorge et al. Europa $\quad \mathrm{Vl}$
$(2011)(9)$

Analisar a comunicação das mães pela primeira vez com os profissionais de saúde e explorar a satisfação, necessidades e expectativas em relação aos serviços de saúde.
0 estudo encontrou alguns pontos de melhoria na comunicação entre as primeiras mães e os profissionais de saúde que poderiam ser levados em consideração nos planos de promoção da satisfação com os serviços de saúde.

2 Vivenciando o cuidado no contexto de uma casa de parto: 0 olhar das usuárias. / MEDLINE/BVS

Gonçalves, Roselane et al. Brasil V (2011) (10)
Compreender a vivência da mulher parturiente no contexto de uma Casa de Parto situada em São Paulo
Os resultados evidenciaram que a mulher que escolhe a Casa de Parto para dar à luz busca pelo cuidado humanizado e que nesse contexto ela passa por experiências positivas e negativas.
Desigualdades sociais e sa-

3 tisfação das mulheres com 0 atendimento ao parto no Brasil: estudo nacional de base hospitalar. / SciELO
d'Orsi,

Eleonora et al. Brasil

(2014) (11)
Identificar fatores associados à avaliação das mulheres quanto à relação profissionais de saúde/parturiente e como esses fatores influenciam a satisfação com o atendimento
As mulheres valorizam a forma como são atendidas pelos profissionais e existem desigualdades de cor, região geográfica e fonte de pagamento do parto nessas relações. ao parto. 
Vivências de mulheres sobre a assistência recebida no processo parturitivo. I SCIELO

Santos, Luciano Marques; Pereira, Samantha Souza da Costa. (2012) (12)

\section{Explorando las emociones} de la mujer en la atención perinatal: Un estudio cualitativo. / SciELO Sánchez, José Espaet al. (2016) nha
Compreender as vivências de puérperas sobre a atenção recebida durante o processo parturitivo em uma maternidade pública de Feira de Santana-Bahia.

A análise de conteúdo e semiológica dos dados demonstrou que as entrevistadas vivenciaram o processo parturitivo com solidão, medo, dor, sofrimento, abandono, e tiveram seus filhos sozinhas.

Explorar as emoç̃es que emergem na mulher durante a gestação, parto e pós-parto ao longo do itinerário assistencial de atenção primária e hospitalar.

\section{- Assistência de enfermagem} durante o trabalho de

Soares Ferreira, Luiza parto e parto: a percepção da mulher. / BDENF

7 Avaliação da satisfação de puérperas em relação ao parto. / BDENF

Satisfação das puérperas

8 atendidas em um centro de parto normal. / BDENF

Parto normal assistido por enfermeira: experiência e satisfação de puérperas. I BDENF

A percepção das puérperas sobre a assistência recebida durante o parto. / BDENF

Validation of the Women's

Views of Birth Labor

11 Satisfaction Questionnaire (WOMBLSQ4) in the Spanish Population. / PUBMED
Perception of childbirth

12 women in Bali, Indonesia: a qualitative study / PUBMED experiences of Japanese

\section{Mairla et al.}

(2017) (14)

Riegert, Isadora Tavares et al. (2018)

Soares, Yndiara Kássia da Cunha et al. (2017) (16)

Freire, Hyanara Sâmea de Sousa et al. (2017) (17)

Sabino, Vanessa Gomes da Rocha Silva et al. (2017)

$$
\text { (18) }
$$

Pozo-Cano,

María Dolores Espaet al. (2020) nha (19) Brasil VI

Investigar a assistência de enfermagem ao trabalho de parto e parto, através da percepção das parturientes.

Analisar a satisfação acerca do trabalho de parto e parto de mulheres que pariram em uma maternidade de alto risco.

Analisar a satisfação das Brasil VI puérperas atendidas em um Centro de Parto Normal.

Descrever a experiência e a satisfação de mulheres que tiveram parto normal assistido por enfermeira.

Apreender a percepção das puérperas sobre 0 atendimento proporcionado durante 0 parto.

Determinar a estrutura fatorial do Questionário de Satisfação do Trabalho de Parto das Mulheres (WOMBLSQ4).
Tanaka, Kazuno et al. Japão VI (2020) (20)
Examinar como os cuidados perinatais prestados são vivenciados por mulheres japonesas em Bali.
Constata-se uma variabilidade emocional devido à coexistência dos modelos tecnocrático e biopsicossocial.

Foram identificadas 16 ideias centrais para

4 perguntas, evidenciando uma relativa

satisfação das parturientes com a assistência de enfermagem prestada.

Constatou-se que houve relação estatística significativa entre a expectativa e a satisfação das mulheres com o parto.

Constatou-se satisfação das puérperas com a assistência recebida, sobretudo pelo apoio contínuo das enfermeiras obstetras.

A enfermeira é reconhecida como profissional diferenciada que fornece apoio físico e emocional e auxilia no relaxamento e no enfrentamento da parturição.

As puérperas apresentaram percepções favoráveis atreladas às atitudes dos profissionais de saúde, à disponibilidade de recursos físicos/materiais e à participação da família.

Na prática clínica, essa escala pode ser relevante para medir os níveis de satisfação durante o parto de mulheres de língua espanhola.

As mulheres japonesas em Bali esperavam cuidados perinatais centrados na mulher e apoio ativo da equipe de enfermagem / obstetrícia durante a gravidez e cuidados pós-natal.

\begin{tabular}{|c|c|c|c|c|c|c|}
\hline 13 & $\begin{array}{l}\text { Maternal and paternal } \\
\text { satisfaction in the delivery } \\
\text { room: a cross-sectional } \\
\text { comparative study. I } \\
\text { PUBMED }\end{array}$ & $\begin{array}{l}\text { Bélanger- } \\
\text {-Lévesque, } \\
\text { Marie-Noelle } \\
\text { et al. (2014) } \\
\text { (21) }\end{array}$ & $\begin{array}{l}\text { Cana- } \\
\text { dá }\end{array}$ & IV & $\begin{array}{l}\text { Avaliar e comparar a satis- } \\
\text { fação de mães e pais com o } \\
\text { parto. }\end{array}$ & $\begin{array}{c}\text { A experiência de nascimento de cada um } \\
\text { dos pais e apoiar os pais em conformidade, } \\
\text { adaptando a prestação de cuidados em torno } \\
\text { do parto. }\end{array}$ \\
\hline 14 & $\begin{array}{l}\text { Perception of women on } \\
\text { the care in the childbirth: } \\
\text { contributions to nursing. } \\
\text { / DOAJ }\end{array}$ & $\begin{array}{l}\text { Brasil, Eysler } \\
\text { Gonçalves } \\
\text { Maia et al. } \\
\text { (2013) (22) }\end{array}$ & Brasil & $\mathrm{VI}$ & $\begin{array}{l}\text { Compreender a percepção das } \\
\text { mulheres quanto à assistência } \\
\text { recebida durante o parto / } \\
\text { nascimento }\end{array}$ & $\begin{array}{l}\text { As mulheres destacaram o bom atendimen- } \\
\text { to recebido, que consiste no carinho e na } \\
\text { dedicação dos profissionais. }\end{array}$ \\
\hline
\end{tabular}


dos artigos encontrados expressa nível VI de evidência, enquanto apenas dois artigos exprimem nível IV de evidência. Os principais achados dispostos nos objetivos e conclusões, estão diretamente associados à satisfação das puérperas em relação à assistência recebida no trabalho de parto e parto nos serviços de saúde (Tabela 1 ).

Dado o exposto foi possível observar que a maioria das puérperas se diz satisfeita com a assistência prestada durante o processo de parto. Motivando ainda a mais a prestação de uma assistência de qualidade, visando o quão importante é este momento diante de todas as expectativas dessas mulheres.

\section{DISCUSSÃO}

A satisfação das mulheres é influenciada pelas expectativas que elas possuem sobre o atendimento que irão receber, não retratando especificamente a qualidade da assistência prestada. (11) No entanto, averiguar a satisfação com o atendimento recebido no trabalho de parto e parto pode ser uma importante ferramenta para a avaliação dos serviços disponíveis e a implementação das boas práticas nas instituições de saúde.

Em concordância com outros estudos, as características do primeiro atendimento recebido no momento da chegada ao hospital foram vitais para a primeira impressão a respeito da instituição. (10) Este e outros estudos apontam que a satisfação está associada à qualidade do relacionamento e comunicação com os profissionais de saúde (respeito, privacidade, atenção e informações recebidas durante o trabaIho de parto/parto, apoio emocional), com a forma como vivenciaram o processo (pouco sofrimento, presença de acompanhante de sua escolha) e com os bons desfechos maternos e neonatais. (11)

É importante destacar também as implicações que o Plano de Parto pode trazer frente à assistência prestada e a satisfação das puérperas com o atendimento recebido. O Plano de Parto e Nascimento é um documento escrito, de caráter legal, em que a mulher expressa seus valores e desejos pessoais para o período pré-natal, parto e pós-parto. (22)

Com base nesse conceito, os profissionais de saúde, ainda no pré-natal, devem utilizar em suas consultas este documento, buscando melhorar a assistência e fortalecer a comunicação da mulher gestante no ambiente hospitalar. Além disso, este é um instrumento de empoderamento feminino, buscando conhecer os desejos e necessidades da mulher naquele momento tão singular que é a gestação. $(23,24)$

Diante da elaboração deste estudo, as limitações da pesquisa referem-se quanto a delimitação de artigos que nem sempre abordavam com exatidão a questão norteadora da pesquisa. Contudo, fica evidente a importância da realização de mais estudos desta natureza para a ampliação da produção de conhecimento e ainda para fomentar a discussão acerca desta temática.

\section{CONCLUSÃO}

Os dados obtidos com a pesquisa apontam para a inexistência de unanimidade quanto à percepção e satisfação das mulheres em relação à assistência ao trabalho de parto e parto, indício de que nem sempre o que é considerado como ideal e recomendado é encarado sob a mesma ótica por todas as mulheres. Sendo esta experiência vivenciada como singular e única para cada muIher. Nesse sentido, atenção, acolhimento e cuidado, assim como o vínculo, são elementos fundamentais para uma assistência mais humanizada e, consequentemente, para o alcance da satisfação destas mulheres em relação à assistência obstétrica recebida.

\section{Referências}

1. Silva IA, Silva PSF, Andrade EWOF, Morais FF, Silva RSS, Oliveira LS Percepção das puérperas acerca da assistência de enfermagem no parto humanizado. Revista Uningá. 2017;53(2):37-43. Disponivel em: http://revista.uninga.br/index.php/uninga/article/view/1440

2. Brasil. Ministério da Saúde. Portaria n. 1.459 de 24 de junho de 2011. Institui, no âmbito do Sistema Único de Saúde, a Rede Cegonha. Diário Oficial da União, Brasilia; 2011.

3. Vitorino de Sousa MP, Alves dos Santos LS, Ferreira Caldas GR, de Assis Moura Batista F, Lopes da Silva CR. Violência obstétrica: fatores desencadeantes e medidas preventivas de enfermagem. Nursing. $2^{\circ}$ de agosto de 2021; 24(279):6015-24. https://doi.org/10.36489/nursing. 2021v24i279p6015-6024

4. World Health Organization. WHO recommendations: intrapartum care for a positive childbirth experience. Geneva: World Health Organization, 2018.
5. Nascimento FCV, Silva MP, Viana MRP. Assistência de enfermagem no parto humanizado. Rev Pre Infec e Saúde [internet]. 2017; 4(6887). Disponível em: http://www.ojs.ufpi.br/index.php/nupcis/article/view/6887

6. Santos MARC, Galvão MGA. A elaboração da pergunta adequada da pesquisa. Rev Resid Pediatr. 2014;4(2):53-56. DOI: htps://doi. org/10.25060/residpediatr

7. Barbosa FT et al. Tutorial para realizar revisão sistemática e meta-análise com estudos de anestesia intervencionista. Rev. Bras. Anestesiol. [Internet]. Junho de 2019;69(3): 299-306. DOI: https://doi.org/10.1016/j. bjane.2019.03.003

8. Melnyk BM.; Fineout-Overholt E. Making the case for evidence-based practice. In B. M. MELNYK \& E. FINEOUT-OVERHOLT. Evidence-based practice in nursing \& healthcare: a guide to best practice. Philadelphia: Lippincot Williams \& Wilkins. 2005; 3-24.

9. Díaz-Sáez J, Catalán-Matamoros D, Fernández-Martínez MM, Grana- 


\section{Referências}

dos-Gámez G. La comunicción e la satisfacción de las primíparas em um servicio público de salud. Gac. Sanit. 2011;25(6): 483-489. DOI: https:// doi.org/10.1016/j.gaceta.2011.03.010

10. Gonçalves R, Aguiar CA, Merighi MAB, Jesus MCP. Vivenciando o cuidado no contexto de uma casa de parto: o olhar das usuárias. Rev Esc Enferm USP. 2011;45(1): 62-70. DOI: https://doi.org/10.1590/S008062342011000100009

11. D'orsi E, Brüggemann OM, Diniz CSG, Aguiar JM, Gusman CR, Torres JA et al. Desigualdades sociais e satisfação das mulheres com o atendimento ao parto no Brasil: estudo nacional de base hospitalar. Cad. Saúde Pública. 2014;30(1): S154-S168. https://doi.org/10.1590/0102-311X00087813

12. Santos LM, Pereira SSC. Vivências de mulheres sobre a assistência. Physis Revista de Saúde Coletiva, Rio de Janeiro. 2012;22(1):77-97. Disponível em: https://www.scielosp.org/article/physis/2012.v22n1/77-97/

13. Arnau Sánchez J, Martínez-Ros MT, Castaño-Molina MA, Nicolás-Vigueras MD, Martínez-Roche ME. Explorando las emociones de la mujer en la atención perinatal. Un estudio cualitativo. Aquichan. 2016; 16(3): 370-381. https://doi.org/10.5294/aqui.2016.16.3.8

14. Soares FLM, Santos ADF, Ramalho RCF, de Araújo AD, Damasceno SS, Figueiredo MFER et al. Assistência de enfermagem durante o trabalho de parto e parto: a percepção da mulher. Rev Cubana Enfermer [Internet]. 2017 Jun [cited 2021 Ago 16]; 33(2). Available from: http://scielo.sld.cu/ scielo.php?script=sci_arttext\&pid=S0864-03192017000200013\&lng=es

15. Riegert IT, Correia MB, Andrade AL, Rocha FNPS, Lopes LGF, Viana APA et al. Avaliação da satisfação de puérperas em relação ao parto. Revista de Enfermagem Ufpe On Line, 2018; 12 (11): 2986. Revista de Enfermagem, UFPE Online. http://doi.org/10.5205/1981-8963-v12i11a23 6863p2986-2993-2018

16. Soares YKC, Melo SSS, Guimarães TMM, Feitosa VC, Gouveia MTO. Satisfação das puérperas atendidas em um centro de parto normal. Rev enferm UFPE on line., [Internet]. Recife, 2017; [cited 2021 Ago 16]; 11(Supl. 11):4563-73. Available from: https://periodicos.ufpe.br/revistas/ revistaenfermagem/article/view/231195/25187

17. Freire HAA, Campos FC, Castro RCMB, Costa CC, Mesquita VJ, Viana RAA. Parto normal assistido por enfermeira: experiência e satisfação de puérperas. Rev enferm UFPE on line., [Internet]. Recife, 2017; [cited 2021 Ago 16]; 11(6):2357-67. Available from: https://periodicos.ufpe.br/revistas/revistaenfermagem/article/view/23398/19057

18. Sabino VGRS, Costa NS, Bracarense CF, Duarte JMG, Simões ALA. A percepção das puérperas sobre a assistência recebida durante o parto. Rev enferm UFPE on line., [Internet]. Recife, 2017; [cited 2021 Ago 16]; 11(10):3913-9. Available from: https://periodicos.ufpe.br/revistas/revistaenfermagem/article/view/25216/24347

19. Pozo-Cano MD, Martín-Salvador A, Pérez-Morente MÁ, Martínez-García E, Luna Del Castillo JD, Gázquez-López M, Fernández-Castillo R, García-García I. Validation of the Women's Views of Birth Labor Satisfaction Questionnaire (WOMBLSQ4) in the Spanish Population. Int J Environ Res Public Health. 2020 Aug 2;17(15):5582. http://doi.org/10.3390/ijerph17155582 PMID: 32748884 ; PMCID: PMC7432014.

20. Tanaka K, Kurniasari NMD, Widyanthini DN, Suariyani NLP, Listyowati R, Urayama A, Wirawan IMA, Yoshimura K. Perception of childbirth experiences of Japanese women in Bali, Indonesia: a qualitative study. BMC Pregnancy Childbirth. 2020 Dec 7;20(1):760. http://doi.org/10.1186/ s12884-020-03466-x PMID: 33287736; PMCID: PMC7720464.

21. Bélanger-Lévesque MN, Pasquier M, Roy-Matton N, Blouin S, Pasquier JC. Maternal and paternal satisfaction in the delivery room: a cross-sectional comparative study. BMJ Open. 2014 Feb 24;4(2):e004013. http:// doi.org/10.1136/bmjopen-2013-004013 PMID: 24566529; PMCID: PMC3939628.

22. Brasil EGM, Queiroz MVO, Fernandes AFC, Costa RF, Xavier EO. Perception of women on the care in the childbirth: contributions to nursing - doi. Acta Scientiarum. Health Sciences, [Internet]. 2013; [cited 2021 Ago 16]; 35(2): 195. http://doi.org/10.4025/actascihealthsci.v35i2.10442 